\title{
Cervicolumbar Coordination in Mammalian Quadrupedal Locomotion: Role of Spinal Thoracic Circuitry and Limb Sensory Inputs
}

\author{
Laurent Juvin, ${ }^{1,2}$ Jean-Patrick Le Gal, ${ }^{1,2}$ John Simmers, ${ }^{1,2}$ and Didier Morin ${ }^{1,2}$ \\ ${ }^{1}$ University Bordeaux, INCIA, UMR 5287, F-33000 Bordeaux, France, and ${ }^{2}$ CNRS, INCIA, UMR 5287, F-33000 Bordeaux, France
}

Effective quadrupedal locomotion requires a close coordination between the spatially distant central pattern generators (CPGs) controlling forelimb and hindlimb movements. Using isolated preparations of the neonatal rat spinal cord, we explore the role of intervening thoracic circuitry in cervicolumbar CPG coordination and the contribution to this remote coupling of limb somatosensory inputs. In preparations activated with bath-applied $N$-methyl-D,L-aspartate, serotonin, and dopamine, the coordination between locomotor-related bursts recorded in cervical and lumbar ventral roots was substantially weakened, although not abolished, when the thoracic segments were selectively withheld from neurochemical stimulation or were exposed to a low $\mathrm{Ca}^{2+}$ solution to block synaptic transmission. Moreover, cervicolumbar CPG coordination was reduced after a thoracic midsagittal section, suggesting that cross-cord projections participate in the anteroposterior coupling. In quiescent preparations, either cyclic or tonic electrical stimulation of low-threshold afferent pathways in C8 or L2 dorsal roots (DRs) could elicit coordinated ventral root bursting at both cervical and lumbar levels via an activation of the underlying CPG networks. When lumbar rhythmogenesis was prevented by local synaptic transmission blockade, L2 DR stimulation could still drive left-right alternating cervical bursting in preparations otherwise exposed to normal bathing medium. In contrast, when the cervical generators were selectively blocked, C8 DR stimulation was unable to activate the lumbar CPGs. Thus, in the newborn rat, anteroposterior limb coordination relies on active burst generation within midcord thoracic circuitry that additionally conveys ascending and weaker descending coupling influences of distant limb proprioceptive inputs to the cervical and lumbar generators, respectively.

\section{Introduction}

During quadruped locomotion, interappendicular coordination is essential to the production of accurate stepping movements, with changes in patterns of coupling between the forelimbs and hindlimbs underlying the expression of different gaits such as walking, trotting, and galloping (Orlovsky et al., 1999). Understanding the neural basis of such adaptive motor function therefore requires knowledge not only of the individual central pattern generator (CPG) networks in the cervical and lumbar spinal cord that produce propulsive movements of the anterior and posterior limbs, respectively (Grillner, 1981; Rossignol, 1996), but also of the neural mechanisms by which the output programs of these spatially distant CPGs are coordinated to ensure effective locomotory behavior.

In principle, the coordination of the rhythmogenic networks controlling the individual limbs could derive from two signaling

Received Sept. 2, 2011; revised Oct. 27, 2011; accepted Nov. 16, 2011.

Author contributions: J.S. and D.M. designed research; L.J., J.-P.L.G., and D.M. performed research; L.J. and J.-P.L.G. analyzed data; L.J., J.S., and D.M. wrote the paper.

This work was supported by doctoral grants from the Demain Debout organization and the Institut pour la Recherche sur la Moelle épinière et I'Encéphale (L.J.). We thank Dr. Didier Le Ray for providing customized scripts for stimulus artifact suppression and electrophysiological signal integration.

Correspondence should be addressed to Dr. Laurent Juvin, Université Bordeaux Segalen, CNRS UMR 5287, Institut des Neurosciences Cognitives et Intégratives d'Aquitaine, Zone Nord, bâtiment 2A, 146 rue Léo Saignat, 33076 Bordeaux, France. E-mail: laurent.juvin@u-bordeaux1.fr.

DOI:10.1523/JNEUROSCI.4640-11.2012

Copyright $\odot 2012$ the authors $\quad 0270-6474 / 12 / 320953-13 \$ 15.00 / 0$ processes: (1) the transfer of coupling information between the cervical and lumbar locomotor CPGs via intercalated intraspinal pathways and (2) coordinating influences that originate extrinsically to the spinal cord, such as peripheral sensory feedback or locomotor-related signals descending from the brainstem. However, despite considerable in vivo data available for the respective roles of propriospinal (Miller et al., 1975; English, 1979; Rossignol et al., 1993) and extraspinal signaling (Matsukawa et al., 1982; Udo et al., 1982; English and Weeks, 1989) in mammalian interlimb coordination, as well as more recent in vitro evidence (Ballion et al., 2001; Juvin et al., 2005; Cowley et al., 2008), the underlying functional pathways and coupling mechanisms remain relatively poorly understood.

The crucial role played by limb sensory feedback in regulating locomotor CPG network operation in the mammalian spinal cord is now widely recognized. Afferent input from hindlimb joint and load receptors to the lumbar generators has long been known to activate treadmill stepping in spinalized cat (Sherrington, 1910; Rossignol et al., 2006; Edgerton et al., 2008) and to control the temporal patterning of ongoing limb movements (Pearson, 2004). Moreover, the major actions of proprioceptive signaling can be reproduced in vitro, where electrical stimulation of hindlimb afferent nerves can elicit locomotor-like output from adjacent, otherwise quiescent lumbar CPG circuitry in the rodent spinal cord (Smith et al., 1988; Whelan et al., 2000; Marchetti et al., 2001) and can reset or entrain lumbar rhythms already in- 

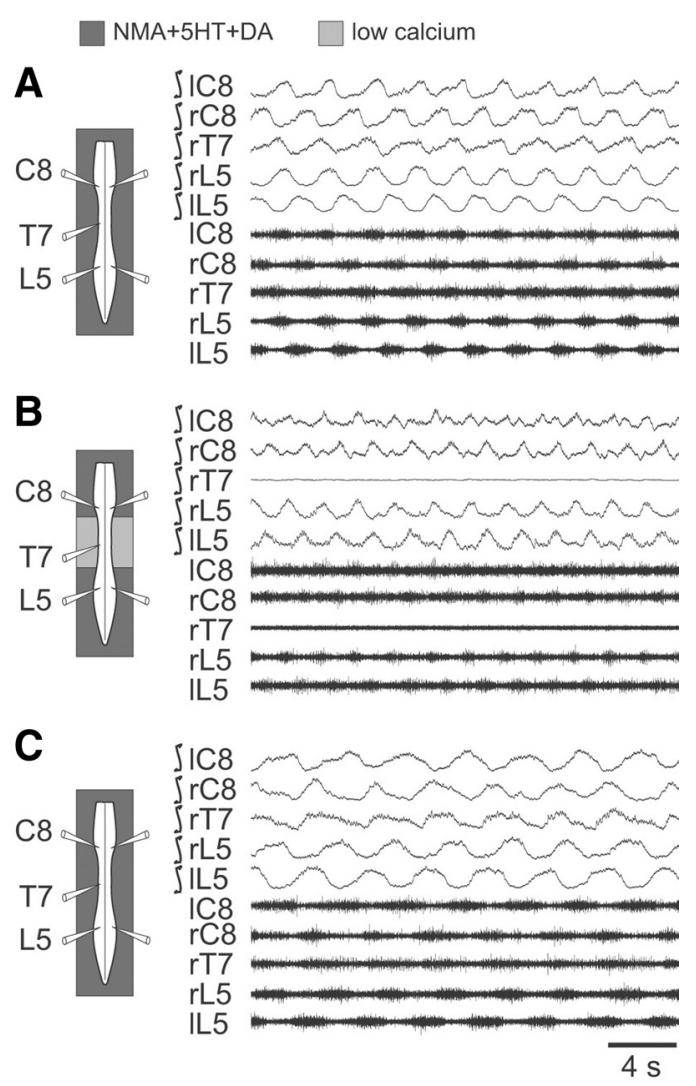

D
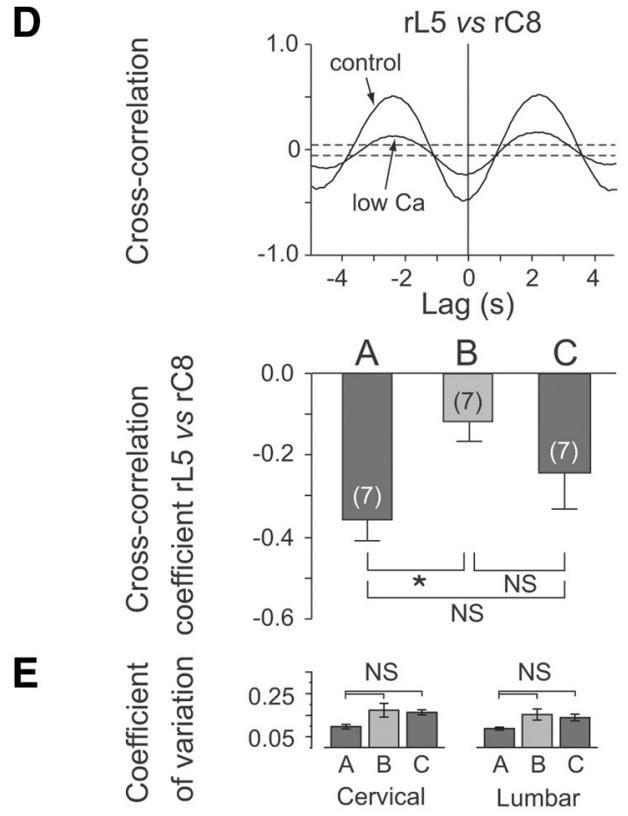

Figure 1. Blockade of synaptic transmission in thoracic segments of the neonatal rat spinal cord weakens the coordination between cervical (forelimb) and lumbar (hindlimb) locomotorrelated rhythmicity. $\boldsymbol{A}-\boldsymbol{C}$, Left, Schematics of the isolated spinal cord with locations of extracellular electrode recordings. Right, Raw extracellular (bottom traces) and corresponding integrated activity (top traces) in left (I) and right ( $\mathrm{r}$ ) cervical (C), thoracic (T) and lumbar (L) ventral roots during fictive locomotion induced by NMA/5-HT/DA applied to the whole cord $(\boldsymbol{A})$, during selective blockade of synaptic transmission in the thoracic cord region (from T3 to T10)

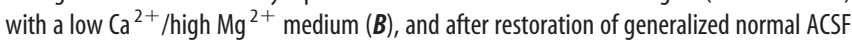
conditions (C). D, Top, Cross-correlograms showing alternation (indicated by a negative crosscorrelation coefficient at lag 0 ) between homolateral lumbar (L5) and cervical (C8) ventral root bursts in control conditions and during exposure of the thoracic segments to low $\mathrm{Ca}^{2+} /$ high $\mathrm{Mg}^{2+}$ (corresponding to $A$ and $\boldsymbol{B}$, respectively). Note that the strength of coordination between L5/C8 motor bursts was reduced under thoracic cord synaptic blockade. Each cross-correlogram duced by neurochemical stimulation (Kiehn et al., 1992; SqalliHoussaini et al., 1993; Iizuka et al., 1997). However, the extent to which limb sensory inputs exert coupling influences on the distant CPGs controlling the other limbs remains unknown.

Here, by making motor root recordings from the isolated newborn rat spinal cord and using split-bath configurations along with pharmacological manipulation and cord lesions, we explore the coordinating interactions between the forelimb/ hindlimb CPGs during locomotor-related rhythmogenesis in the absence of supraspinal influences and under conditions where limb afferent input activation can be experimentally controlled. Our results indicate that the orchestration of coordinated quadrupedal stepping patterns is accomplished at least in part by the cooperative action of intrinsically active propriospinal circuitry that links the forelimb/hindlimb generators and the distributed central influences of extrinsically arising proprioceptive signals resulting from limb movements.

\section{Materials and Methods}

In vitro spinal cord preparations. All procedures were conducted in accordance with the local ethics committee of the University of Bordeaux 2 and the European Communities Council Directive. Experiments were performed on newborn ( 0 to $3 \mathrm{~d}$ old $)$ Wistar rats that were obtained from timed pregnant females raised in our laboratory's breeding facility. Animals were deeply anesthetized by hypothermia and decapitated. The skin and muscles were rapidly removed and preparations were then placed in a $25 \mathrm{ml}$ recording chamber containing circulating artificial CSF (ACSF; see composition below) maintained at $10^{\circ} \mathrm{C}$. The flow rate was set so as to change the total chamber volume within $5 \mathrm{~min}$. The dissection was then continued under a binocular microscope to gently isolate the spinal cord with its dorsal and ventral roots still attached. The cord was then fixed on a Sylgard resin block with the ventral surface upward. Preparations were superfused continuously with ACSF equilibrated with $95 \% \mathrm{O}_{2} / 5 \% \mathrm{CO}_{2}$, $\mathrm{pH}$ 7.4, and containing the following (in $\mathrm{mm}$ ): $113 \mathrm{NaCl}, 4.5 \mathrm{KCl}, 1$ $\mathrm{NaH}_{2} \mathrm{PO}_{4}, 2 \mathrm{CaCl}_{2}, 1 \mathrm{Mg} \mathrm{Cl}_{2}, 25 \mathrm{NaHCO}_{3}$, and 11 D-glucose. At the end of the dissection, the temperature of the bathing medium was progressively raised to $22-25^{\circ} \mathrm{C}$ before recordings began.

Extracellular recordings and electrical stimulations. Motor output activity in spinal ventral roots was recorded using glass suction electrodes. Signals were amplified by homemade AC amplifiers $(10,000 \times)$, bandpass filtered $(0.1-3 \mathrm{kHz}$ ), rectified, integrated (sample rate, $3 \mathrm{kHz} ; \tau=20$ $\mathrm{ms}$ ), digitized, and stored on a computer hard disk (Spike2 software; Cambridge Electronic Design) for off-line analysis.

Electrical pulse train stimulation over an amplitude range of 0.2 to $1 \mathrm{~V}$ at $0.3 \mathrm{~ms}$ per pulse and $1-5 \mathrm{~Hz}$ was applied to spinal dorsal roots (DRs) via glass suction electrodes using an eight-channel digital stimulator (A.M.P.I.). The stimulation followed a procedure described previously (Morin and Viala, 2002; Giraudin et al., 2008), which was based on this study's finding that the activation of large-diameter, and therefore presumed proprioceptive, DR axons in isolated in vitro preparations requires relatively low stimulus intensities. The classification of activated DR afferents as "low threshold" derived from experiments where a proximally placed recording electrode was used to detect the threshold (T) for en passant $\mathrm{DR}$ impulses elicited by a more distal afferent nerve stimulation (see Fig. 8 A). Unless otherwise stated in the text, a value of 1.5 times this threshold stimulus intensity was applied to a given DR throughout the course of the experiment.

$\leftarrow$

was computed from 2-3 min of activity; dashed lines indicate the $95 \%$ ( \pm 2 SEM) confidence interval. Bottom, Histograms showing the mean cross-correlation coefficients ( \pm SEM) of homolateral $L 5$ versus $C 8$ ventral root bursts in the three experimental conditions illustrated in $A-C$. Numbers of preparations are indicated in parentheses. $\boldsymbol{E}$, Histograms showing mean coefficients of variation (vertical bars) and corresponding SDs (vertical lines) of cervical (left) and


the thoracic cord region. ${ }^{*} p<0.05$. NS, Not significantly different. 
Drug application. Pharmacological substances were bath applied by means of gravity supply, beginning at a minimum of $30 \mathrm{~min}$ after the end of dissection. A mixture of $N$-methyl-D,L-aspartate (NMA; 5-20 $\mu \mathrm{M}$ ), serotonin $(5-\mathrm{HT} ; 10 \mu \mathrm{M})$, and dopamine (DA; $100 \mu \mathrm{M}$ ) (all purchased from Sigma) was used to elicit prolonged and stable episodes of fictive locomotion (Juvin et al., 2005). In some experiments, the recording chamber was partitioned into two or three compartments with barriers of syringe-ejected Vaseline to allow the differential exposure of selected spinal cord regions to pharmacological stimulation. In other cases, a modified ACSF containing a lowered $\mathrm{Ca}^{2+}$ concentration $(0.1 \mathrm{~mm}$ $\mathrm{CaCl}_{2}, 5 \mathrm{mM} \mathrm{MgCl}_{2}$ ) was bath-applied to either the thoracic, lumbar, or cervical compartments to reversibly block all chemical synaptic transmission within the segments of that cord region (see Fig. $8 \mathrm{~A}$ ). The water tightness of partitions was checked at the end of the experiment by observing the movements of methylene blue added to the bathing medium on one side of the Vaseline bridge.

Cord lesions. In an additional series of experiments, the two sides of the thoracic cord from segments T3 to T10 were separated by making a sagittal midline split using a razor blade fragment. Again a control period of $30 \mathrm{~min}$ was respected before recording data collection.

Data analysis. The coordination between the burst activities of different spinal ventral roots was examined by means of cross-correlation analysis of at least $120 \mathrm{~s}$ of stable fictive locomotory-related activity. The resultant correlograms were plotted with 95\% (means \pm 2 SEM) confidence intervals that were calculated using Statistica 6 software (Statsoft). Cross-correlation coefficients between two locomotor activities allowed estimation of their coupling strengths, whereby values approaching 1 indicated synchrony, whereas values toward -1 corresponded to burst alternation. In the same experiments (Figs. 1,2), the regularity of cervical and lumbar rhythms was determined by measuring the mean locomotor cycle period before, during, and after a given pharmacological manipulation, and by calculating the mean coefficients of variation, which in each case was defined as the ratio of the SD to the mean.

The phase relationship between locomotor bursts in two different ventral roots was determined by constructing a circular phase diagram from normalized cycles (Kjaerulff and Kiehn, 1996). In each experiment, 15 bursts of a given ventral root (VR1) were randomly selected from a sequence of stable rhythmic activity and taken as the reference. The phase values $(\Phi)$ of burst onsets in a second ventral root (VR2) with respect to VR1 were determined by dividing the latency from the start of each VR1 burst to the next VR2 burst onset by the VR1 cycle period. The phase values were then plotted on a circular phase diagram with a scale ranging from 0 to 1 , where values close to 0.5 reflected burst alternation, and values approaching 0 or 1 indicated phase coincidence. This provided a mean vector $(\mu)$ whose direction and length ( $r$, also on a scale of 0 to 1$)$ indicated, respectively, the preferred phase and strength of phase coupling between bursts in the selected nerve pairs. The evaluation of $r$, using the Rayleigh test to determine the concentration of phase values around the mean vector, estimated the coupling strength (considered to be significant for a probability of $p<0.05$ ).

Group values were expressed as means \pm SEM. Differences between means were analyzed using a statistical software package (Sigma Stat for Windows; SPSS) and assessed by one way ANOVA with a Tukey posttest. Differences in mean values for each parameter were taken to be significant at $p<0.05$.

\section{Results}

Active participation of thoracic circuitry in cervicolumbar CPG coordination

As reported previously (Barriere et al., 2004; Juvin et al., 2005), the bath application of a combination of NMA $(10 \mu \mathrm{M}), 5-\mathrm{HT}(10$ $\mu \mathrm{M})$, and DA $(100 \mu \mathrm{M})$ to in vitro spinal cord preparations elicits prolonged episodes of stable fictive locomotion (Fig. $1 \mathrm{~A}$ ). Simultaneous extracellular recordings from left and right cervical (C8) and lumbar (L5) ventral roots that primarily innervate extensor muscles of the forelimbs (McKenna et al., 2000) and hindlimbs (Kjaerulff and Kiehn, 1996), respectively, revealed closely coordinated rhythmic activity [e.g., cross-correlation coefficient $(\rho)$
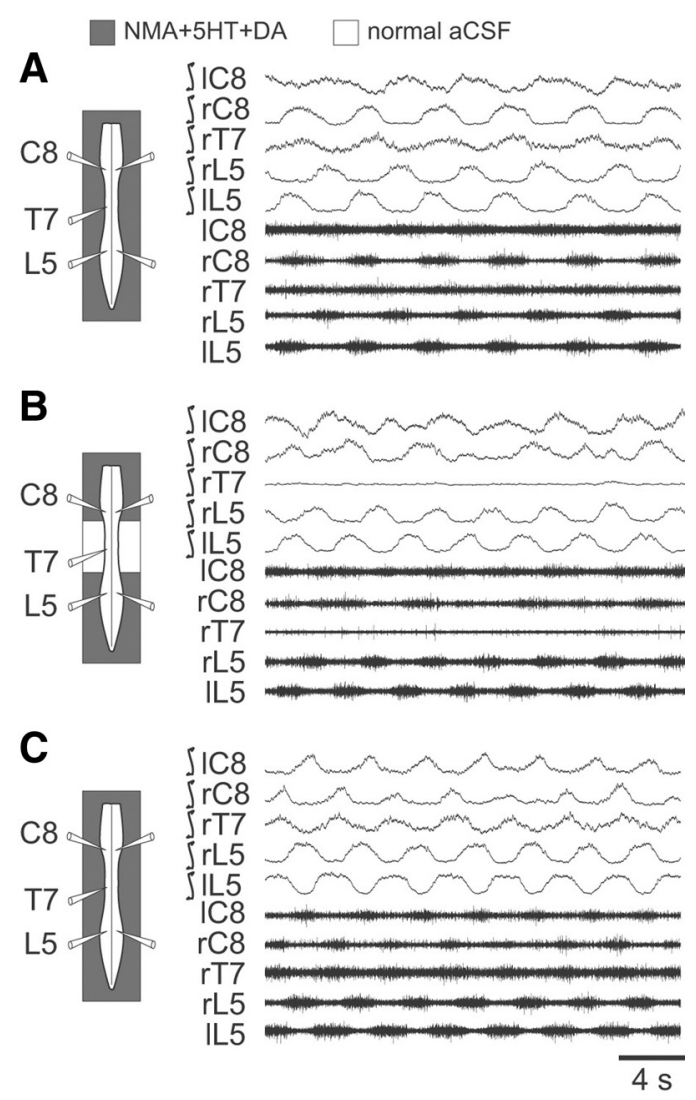

D

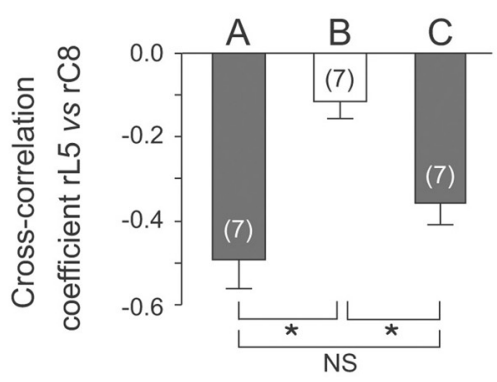

E

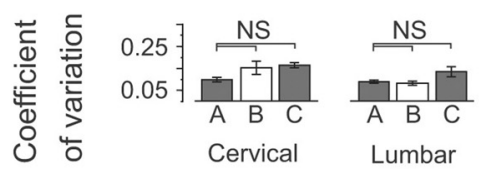

Figure 2. Neurochemical activation of the thoracic cord region significantly enhances the coordination of cervicolumbar locomotor rhythmicity. $A-C$, Left, Schematics of experimental procedure. Right, Integrated and corresponding raw extracellular recordings from bilateral cervical (C), thoracic $(T)$, and lumbar $(\mathrm{L})$ ventral roots during bath application of NMA/5-HT/DA to the whole cord $(\boldsymbol{A})$, to the cervical and the lumbar cord enlargements only $(\boldsymbol{B})$, and again to the entire cord $(\boldsymbol{C})$. In $\boldsymbol{B}$, thoracic segments from T3 to T10 were exposed to normal ACSF. D, Mean cross-correlation coefficients ( \pm SEM) of right lumbar ( $r L 5)$ versus right cervical $(r C 8)$ ventral root activity during NMA/5-HT/DA application to the whole cord $(A, C)$ or to cervical and lumbar spinal segments with the thoracic cord under normal ACSF $(\boldsymbol{B})$. $\boldsymbol{E}$, Histograms of the mean coefficients of variation (vertical bars) and corresponding SDs (vertical lines) of cervical (left) and lumbar rhythms (right) in the three experimental conditions. Numbers of preparations are indicated in parentheses. ${ }^{*} p<0.05$. NS, Not significantly different.

for bursts in right L5 vs right $\mathrm{C} 8,-0.36 \pm 0.05 ; n=7$ preparations; Fig $1 D$, cross-correlogram, bottom left histogram bar] that alternated both bilaterally at cervical and lumbar levels (mean phase value, $\Phi=0.48 ; n=35$ preparations) and longitudinally in the extensor (C8 and L5) motor roots of each side $(\Phi=0.61 ; n=$ $35)$. This coupled cervicolumbar burst pattern, which corre- 
sponded to an interlimb coordination appropriate for in vivo quadrupedal walking (Orlovsky et al., 1999), was also associated with rhythmic bursting activity in the ventral roots of midcord thoracic segments (Fig. 1A, T7 trace).

In principle, the strict coordination between the remote lumbar and cervical pattern generators could be accomplished by direct, long-projecting propriospinal pathways or indirectly by coupling signals that are relayed synaptically through the interposed thoracic segments. Indeed, separate neuroanatomical (Reed et al., 2006) and electrophysiological evidence (Cazalets, 2005; Juvin et al., 2005) has suggested that both transthoracic pathways and local circuit interactions contribute to lumbocervical coupling in the rat spinal cord. On this basis, we argued that a selective blockade of synaptic transmission in the thoracic cord region should prevent any cervicolumbar coordination by relay signaling without disrupting the coupling action of any through-projecting axonal pathways. As seen in Figure $1 B$, during continued chemical activation of the cervical and lumbar CPGs, a local exposure of the midcord region (from T3 to T10) to a low $\mathrm{Ca}^{2+} /$ high $\mathrm{Mg}^{2+}$ ACSF blocked activity in the thoracic segments (T7 trace) and significantly weakened $(\rho=-0.12 \pm$ $0.04 ; p=0.025 ; n=7$ preparations) the coupling between the ongoing cervical and lumbar rhythms (Fig. $1 D$, middle histogram bar). Although this reduction in cervicolumbar coordination coincided with a decrease in thoracic cord activation, it is possible that the coupling impairment was also associated with a disorganization of rhythm production in one or both of the spinal enlargements. To test this latter possibility, the regularity of cervical and lumbar rhythmicity was assessed before, during, and after the exposure of the thoracic segments to the low $\mathrm{Ca}^{2+} /$ high $\mathrm{Mg}^{2+}$ ACSF. As illustrated in Figure $1 E$, no significant differences in the coefficients of variation were observed in the three experimental conditions, indicating that the temporal organization of neither the ongoing cervical nor lumbar rhythms was perturbed by the thoracic low $\mathrm{Ca}^{2+}$ exposure. Finally, the original level of anteroposterior coordination was partially restored after removing the thoracic synaptic blockade by washout with normal NMA/5-HT/DA-containing medium (Fig. 1C,D). These results therefore confirmed the importance of synaptically relayed activity through thoracic spinal circuitry for forelimb and hindlimb CPG coordination.

In a previous study, Ballion et al. (2001) reported that the pharmacological activation of either the lumbar or cervical generators could elicit correlated burst activity in the thoracic segments, leading to the suggestion that passively distributed ascending and descending signals from the two cord enlarge-

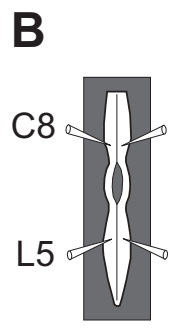

C


rL5 vs IL5

Figure 3. Thoracic cross-cord connections contribute to the coordination of cervicolumbar locomotor rhythmicity. $A, B$, Left,

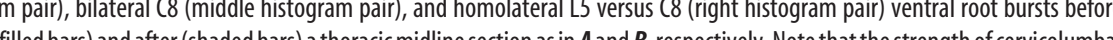
pathways. Numbers of preparations are indicated in parentheses. ${ }^{*} p<0.05$. NS, Not significantly different.

ments are sufficient to maintain cervicolumbar coordination. To test this possibility in a further series of split-bath experiments, we activated the cervical and lumbar CPGs with NMA/5-HT/DA while locally applying standard ACSF to the thoracic cord region. In initial control conditions, when the activating mixture was applied to the entire spinal cord, cervical (C8), thoracic (T7) and lumbar (L5) ventral roots displayed typical locomotory-related burst activity (Fig. 2A) that, as seen previously, was tightly coordinated in a single regular pattern (e.g., right L5 versus right C8, $\rho=-0.49 \pm 0.07 ; n=7$ preparations; Fig. $2 D$, left bar). However, when the thoracic segments were selectively exposed to normal ACSF, the cervical and lumbar ventral roots continued to express robust rhythmic bursting, but the thoracic cord region fell silent and now the coordination between the anterior and 

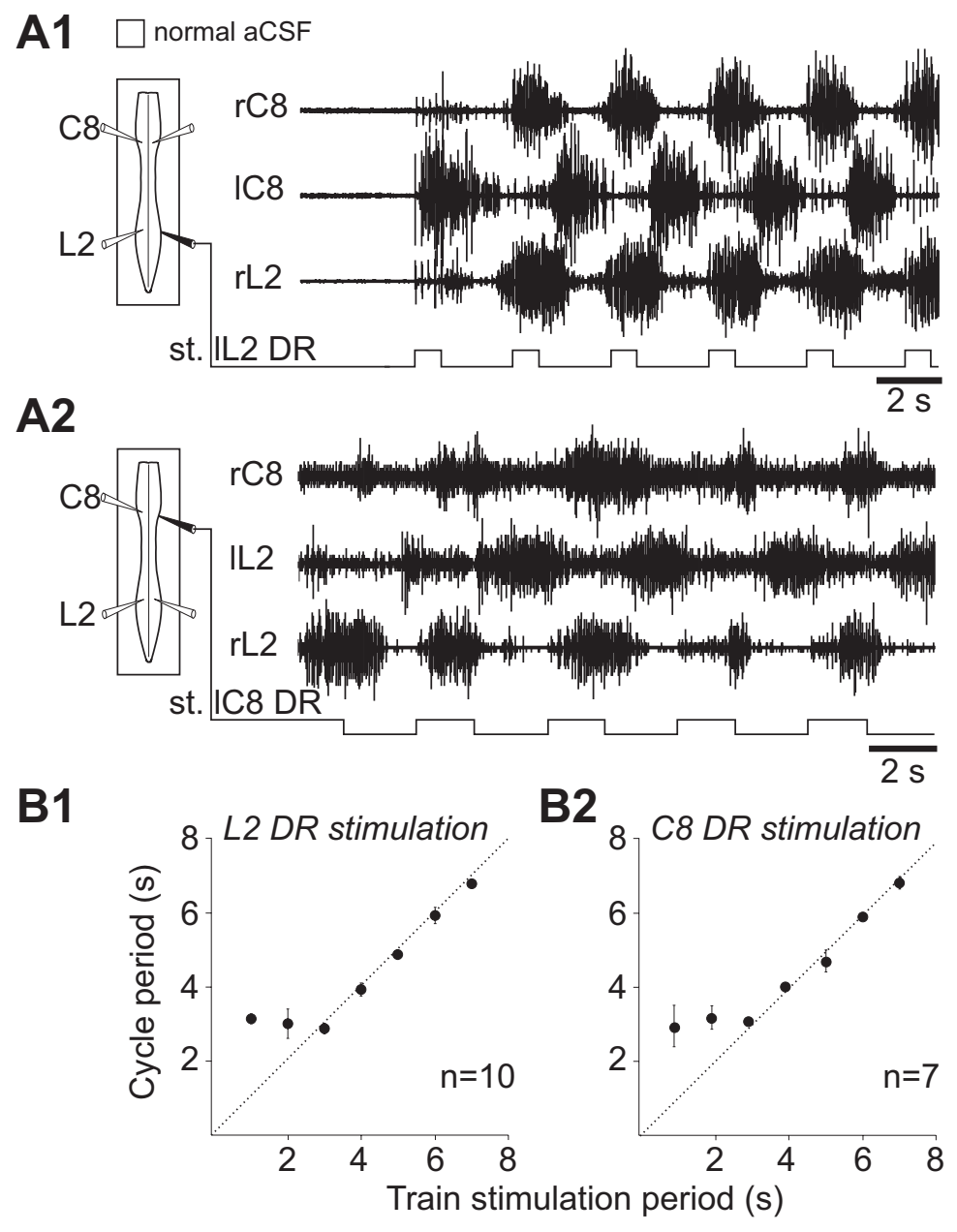

Figure 4. Cyclic activation of low-threshold lumbar or cervical DR afferent fibers elicits coordinated cervicolumbar locomotor bursting. $A 1, A 2$, Left, Schematics indicating the positions of ventral root recording (unfilled) and DR stimulating (filled) electrodes. Right, Raw ventral root activity at cervical (C8) and lumbar (L2) levels in response to electrical train stimulation (st.) of lumbar (L2; A1) or cervical (C8; $A 2)$ DRs. B1, B2, Scatter plots of the relationship between the cycle period of rhythmic motor bursts and the period of lumbar (B1) or cervical (B2) DR stimulus trains. Each point represents the mean motor burst period during 5-10 consecutive cycles of DR stimulation. The dashed lines in $\mathbf{B} 1$ and $\mathbf{B 2}$ indicate a one-to-one coupling, which in both cases fails with stimulus periods below $\sim 3 \mathrm{~s}$.

posterior CPG rhythms was significantly decreased $(\rho=-0.11 \pm$ $0.04 ; p<0.001 ; n=7$ preparations; Fig. $2 D$, middle bar, compare control bar, left). Here again, the absence of significant changes in the coefficient of variation of cervical and lumbar rhythmicity during this thoracic ACSF manipulation (Fig. $2 E$ ) suggested that the decrease in cervicolumbar coupling was not additionally associated with a disorganization of rhythm production at either spinal levels. Finally, strongly coordinated cervicolumbar activity in association with thoracic ventral root bursting was restored when the midcord region was again bathed in the modulatorcontaining medium (Fig. $2 C, D$, right bar). These findings were therefore consistent with the conclusion that the intervening midthoracic segments contribute actively to inter-CPG coupling via intrinsic network mechanisms that presumably, like those of the cervical and lumbar generators, are activated when their levels of excitability are sufficiently elevated, as occurs during direct exposure to NMA/5-HT/DA.

Involvement of thoracic cross-cord projections in cervicolumbar coordination

Recent evidence has indicated that commissural connectivity in the thoracic segments contributes to the descending transmission of brainstem locomotor command signals to the lumbar generators (Cowley et al., 2009), raising the possibility that similar cross-cord propriospinal pathways might also intervene in cervicolumbar CPG coordination. To assess this possibility, we performed a midsagittal section of the thoracic spinal cord in isolated preparations $(n=7)$ under whole-cord activation by NMA/5HT/DA. As seen previously (Fig. 2), in the control intact cord, bilateral lumbar extensor burst activity occurred in strict alternation $(\rho=-0.75 \pm 0.02$; Fig. $3 A, C$, left, left histogram bar) as did left-right bursting in the cervical extensor counterparts $(\rho=$ $-0.55 \pm 0.06$; Fig. $3 A, C$, middle, left bar). Similarly, activity in homolateral cervical and lumbar extensor ventral roots was also closely coordinated in an out-of-phase relationship $(\rho=-0.81 \pm 0.02$; Fig. $3 A, C$, right, left bar). Under continued chemical activation, but after a midsagittal section from T3 to T10, the alternating left-right rhythms of the lumbar and cervical generators were not significantly altered $(\rho=-0.75 \pm 0.04, p=0.89$ and $\rho=$ $-0.31 \pm 0.15, p=0.11$, respectively; Fig. $3 B, C$, left, middle panels, right histogram bars), whereas the strength of longitudinal coupling between the cervical and lumbar rhythms was now significantly decreased $(\rho=-0.62 \pm 0.07 ; p=0.045$; Fig. $3 B, C$, right, right bar). Together, therefore, the above findings strongly support the conclusion that the maintenance of wellcoordinated anteroposterior rhythmogenesis in the neonatal rat spinal cord requires the active propagation of coupling signals through thoracic circuitry and the involvement, at least in part, of midline-crossing segmental pathways.

\section{Implication of proprioceptive afferent feedback in} cervicolumbar coupling

In addition to the involvement of propriospinal circuitry in cervicolumbar CPG coupling, to what extent might extrinsic input signals from limb proprioceptors contribute to the coordination of locomotory limb movements? To address this question, lowthreshold hindlimb and forelimb afferent pathways that are presumed to originate from muscle and joint receptors (Kudo and Yamada, 1987; Morin and Viala, 2002) were rhythmically activated by applying electrical stimuli (1-7 s pulse trains at a pulse intensity, duration, and frequency of 1.5 times threshold; $0.3 \mathrm{~ms}$ and $1-5 \mathrm{~Hz}$, respectively) to a lumbar L2 or cervical C8 DR in otherwise quiescent spinal cord preparations under normal ACSF exposure. As seen in Figure 4A1, the onset of such a stimulation of a lumbar L2 DR elicited strongly coordinated burst activity at the posterior and anterior cord levels in a characteristic fictive walking pattern that consisted of left/right alternation in homologous ventral roots (right/left C8) and simultaneous bursting in homolateral (C8 and L2) ventral roots. Moreover, the DR stimulus-induced motor rhythm could be entrained in a oneto-one coupling over a range of cycle periods (from $3-7 \mathrm{~s} ; n=10$ 
preparations; Fig. 4B1), although with train periods inferior to $3 \mathrm{~s}(1-2 \mathrm{~s})$, the same afferent stimulation continued to evoke coordinated cervicolumbar bursting but was unable to impose full one-to-one coupling (Fig. 4B1). Similarly, rhythmical electrical stimulation of a cervical C8 DR induced coordinated cervicolumbar locomotor-like bursting (Fig. 4A2). Here again, this distributed motor pattern, which was less robust than that elicited by L2 DR stimulation, could only be entrained in a one-to-one coupling at train stimulus periods $\geq 3 \mathrm{~s}$ ( $n=7$ preparations; Fig. 4 B2).

Although these results clearly show that an activation of either forelimb or hindlimb sensory pathways can induce coordinated bursting activity at the cervical and lumbar enlargements, they do not indicate whether the elicited pattern derives from an active intervention of the limb CPGs themselves. Indeed, the possibility that the stimulus-evoked patterns could simply result from a "reflex" activation of premotor interneurons and/or motoneurons (Sherrington, 1910; Sherrington, 1917), without the involvement of central rhythmogenic circuitry, could not be totally excluded. However, several lines of evidence argue against this possibility. First, a purely reflexive activation of limb motoneurons would result in a strict one-to-one coupling entrainment of their discharge regardless of the stimulus train period, which was not observed in our experiments where one-to-one coupling coupling failed at relatively low-stimulus cycle periods (Fig. 4B1,B2). Second, in $80 \%$ ( 8 of 10 ) of the preparations in this experimental series, locomotor-like burst discharge continued to be expressed in both cervical and lumbar motor roots after an episode of lumbar L2 DR stimulation had ceased. In the majority of such cases, this poststimulus activity consisted of one or two additional bursts, but in three episodes from different preparations (Fig. 5A) it comprised five or more wellcoordinated cycles. Again, such persistent bursting in the remote output nerves does not readily comply with a simple reflex activating process. Third, in the same in vitro preparations, tonic electrical DR stimulation could elicit coordinated locomotor-like activity, and therefore in a rhythmic pattern that was completely unrelated to the timing of afferent nerve stimulation. This is illustrated in Figure $5 B$, where repetitive, constant frequency stimulation of a L2 DR (pulse intensity, 1.5 times T; duration, 0.3 $\mathrm{ms}$; frequency, $3 \mathrm{~Hz}$ ) led to coordinated ventral root bursting that lasted 5 to $10 \mathrm{cy}$ cles in a previously silent spinal cord $(n=5$
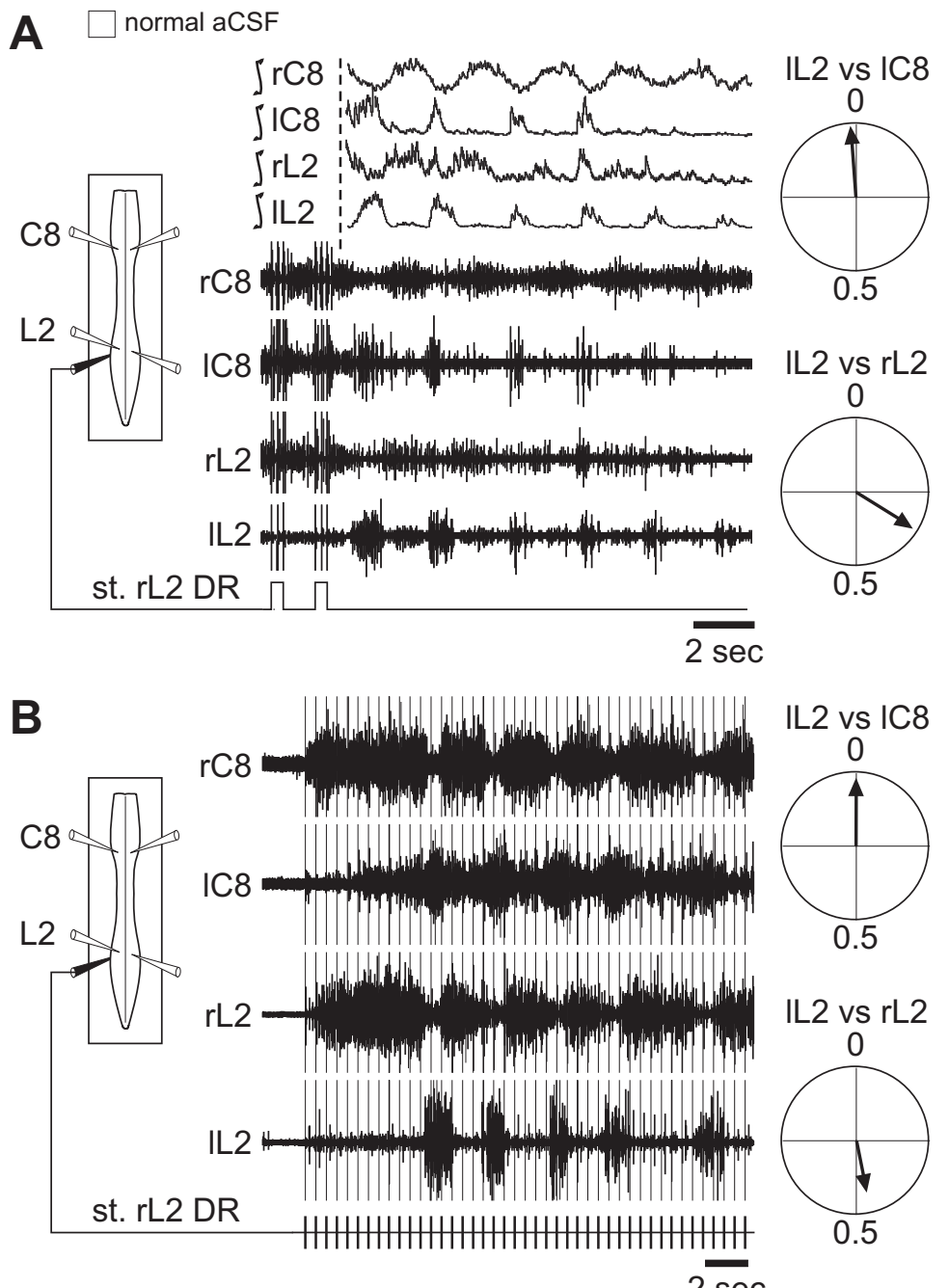

IL2 vs IC8

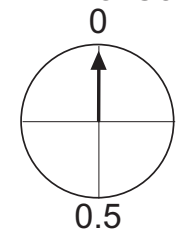

IL2 vs rL2
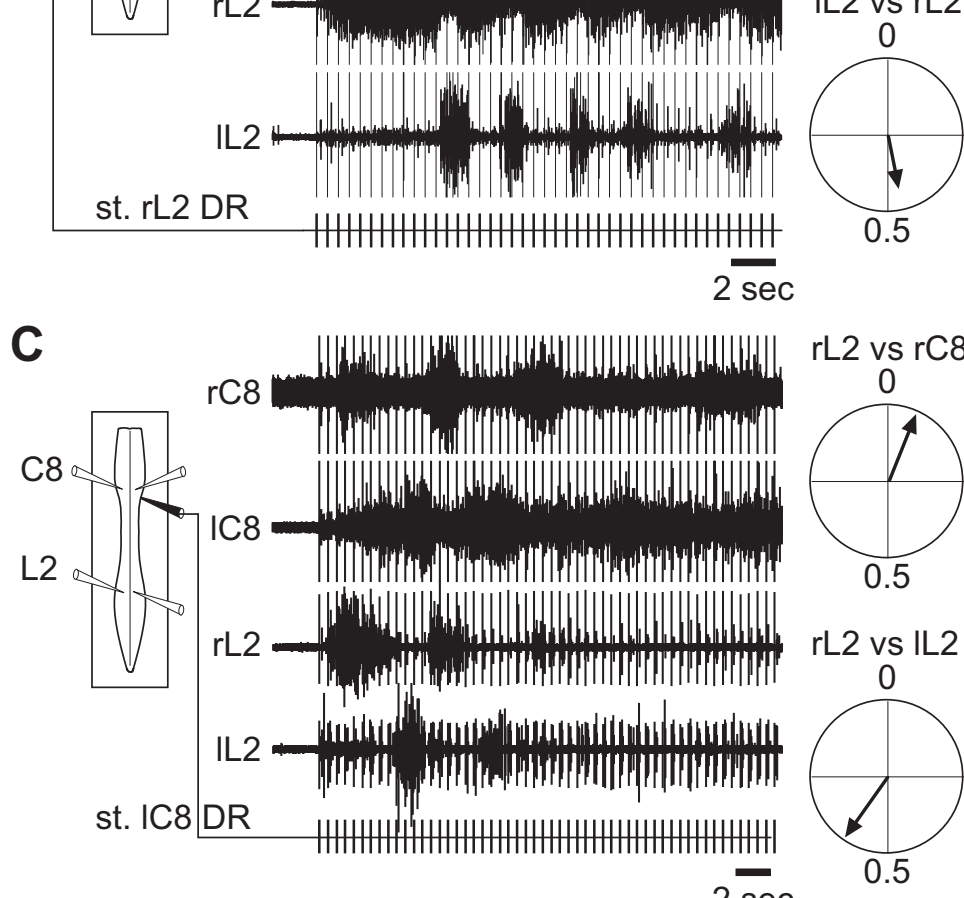

rL 2 vs rC8
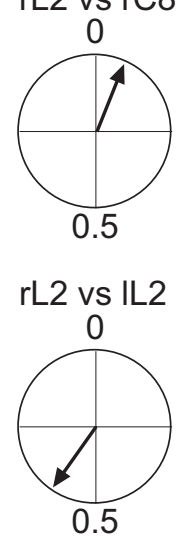

Figure 5. The DR stimulation-elicited motor patterns result from the activation of locomotor rhythm-generating circuitry. $\boldsymbol{A}$, Left, Schematic of the preparation with recording electrodes (unfilled) placed on bilateral cervical (C8) and lumbar (L2) ventral roots and a stimulating electrode (filled) on right L2 DR. Middle, Integrated and raw motor activity immediately after the termination of an episode of cyclic L2 DR stimulation (st.). Note the occurrence of several cycles of locomotor-like bursting after termination of afferent input stimulation. Right, Circular plots (from 15 poststimulus cycles in 3 preparations) of the phase relationships between motor burst onsets in ipsilateral left $L 2$ versus $C 8$ (top) and left $L 2$ vs right $L 2$ (bottom) ventral roots. $B, C$, Left, Schematic of the experimental procedure, which was the same as in $\boldsymbol{A}$ except that the right L2 (B) or the left C8 (C) DR was now stimulated tonically (bottom traces). In both cases, well-coordinated cervicolumbar ventral root bursting was expressed in a pattern that was unrelated to the timing of DR stimulation. Right, Corresponding phase plots of L2 versus ( 8 (top) and left versus right $L 2$ (bottom) ventral root bursts in the two tonic stimulus conditions. The plots in $\boldsymbol{B}$ and $\boldsymbol{C}$ were each constructed from 15 cycles expressed during stimulation-induced bursting in five and three different preparations, respectively. 
A1 Pharmacological activation

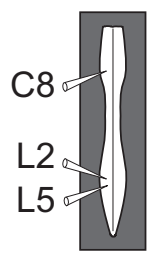

A2
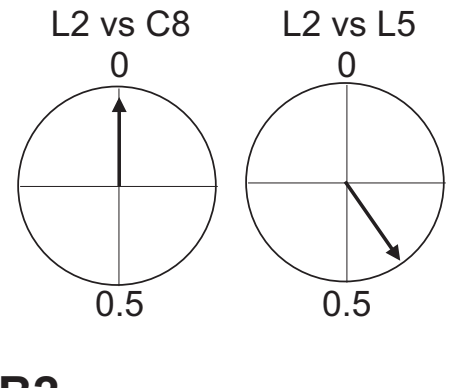

B2
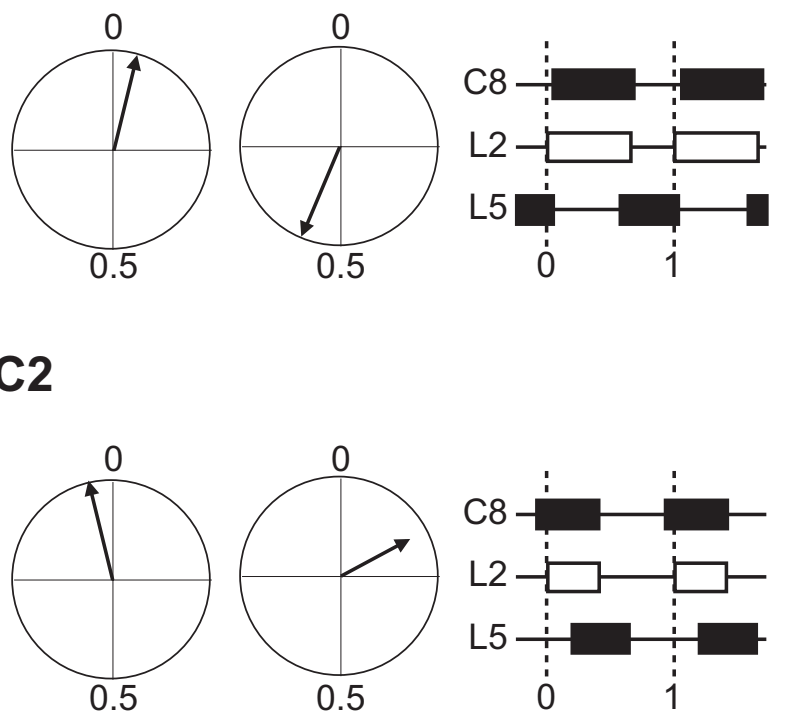

fictive extension fictive flexion

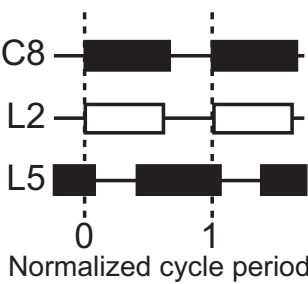

C2

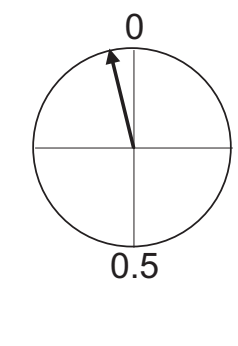

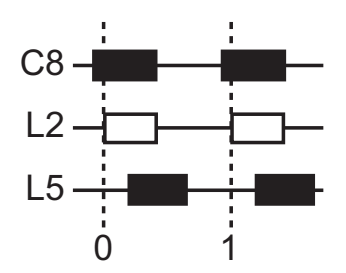

\section{B1 Lumbar DR stimulation}



Figure 6. Comparison of fictive locomotor patterns evoked either by neuroactive drug application or by electrical stimulation (st.) of low-threshold limb sensory pathways. A1, B1, C1, Left, Schematics of experimental procedure. Recording electrodes (unfilled) were placed on ipsilateral cervical (C8) and lumbar (L2, L5) ventral roots. Right, Typical coordinated motor burst patterns induced by NMA/5-HT/DA application to the whole cord (A1) or by rhythmic electrical train stimulation (filled electrode) of ipsilateral lumbar (L2; B1) or cervical (C8; C1) DRs under normal ACSF exposure. A2, B2, C2, Left, Corresponding circular plots of the phase relationships between the onsets of motor bursts recorded over $\geq 15$ random cycles from L2 and C8 (left plots) or L2 and L5 (right plots) ventral roots in the three experimental conditions. Right, Phase diagrams showing duty cycles of ipsilateral $C 8, \mathrm{~L} 2$, and $L 5$ ventral root bursts during normalized cycles of fictive locomotion in each case.

preparations). Furthermore, in three preparations, a similar induction of cervicolumbar rhythmicity, comprising two to six burst cycles, occurred in response to tonic stimulation of a C8 DR (Fig. 5C). Finally, in all three experimental conditions illustrated in Figure 5, the burst-phase relationships corresponded globally to quadrupedal fictive walking, whereby homolateral lumbar (flexor) L2 and cervical (extensor) C8 motoneurons were synchronously active while bursting in bilateral L2 and C8 homologues occurred in alternation (Fig. 5 , circular diagrams, right). Together, these findings therefore indicate that inputs from forelimb or hindlimb proprioceptive pathways are capable of eliciting coordinated locomotor-related output patterns via an activation of the central rhythmogenic circuits in both the cervical and lumbar regions of the isolated rat spinal cord.

\section{Influence of limb sensory inputs on motor burst-phase relationships}

Although the above findings indicated that the activation of individual lumbar or cervical afferent pathways can trigger coordinated cervical and lumbar CPG operation, whether this input information can also shape the temporal organization of the dis- tant motor patterns produced remained unclear. To explore this issue, we compared the phase relationships between ipsilateral flexor (L2) and extensor (L5 and C8) motor root bursts during episodes of fictive locomotion evoked either pharmacologically by NMA/5-HT/DA application ( $n=35$ preparations; Fig. $6 A 1)$ or by cyclic electrical stimulation of a lumbar L2 $(n=8$; Fig. $6 \mathrm{B1})$ or cervical C8 ( $n=9$; Fig. $6 C 1)$ dorsal root. All burst-phase relationships were expressed in reference to motor discharge in $\mathrm{L} 2$, as this is typically the most stable ventral root activity during fictive locomotion produced by the newborn rat spinal cord (Falgairolle and Cazalets, 2007).

Regardless of the method of locomotor CPG activation, bursts in cervical C8 and lumbar L2 motoneurons were always simultaneously expressed (Fig. 6A2,B2,C2, left, corresponding circular phase plots of burst onsets; right, normalized phase diagrams) in accordance with a fundamental feature of quadrupedal walking (Juvin et al., 2005). Similarly, the typical antiphase relationship occurring between homolateral lumbar extensor (L5) and flexor (L2) motor bursts during chemical cord activation (Fig. 6A2, right polar plot, phase diagram; $\Phi=0.40$ ) was also preserved 

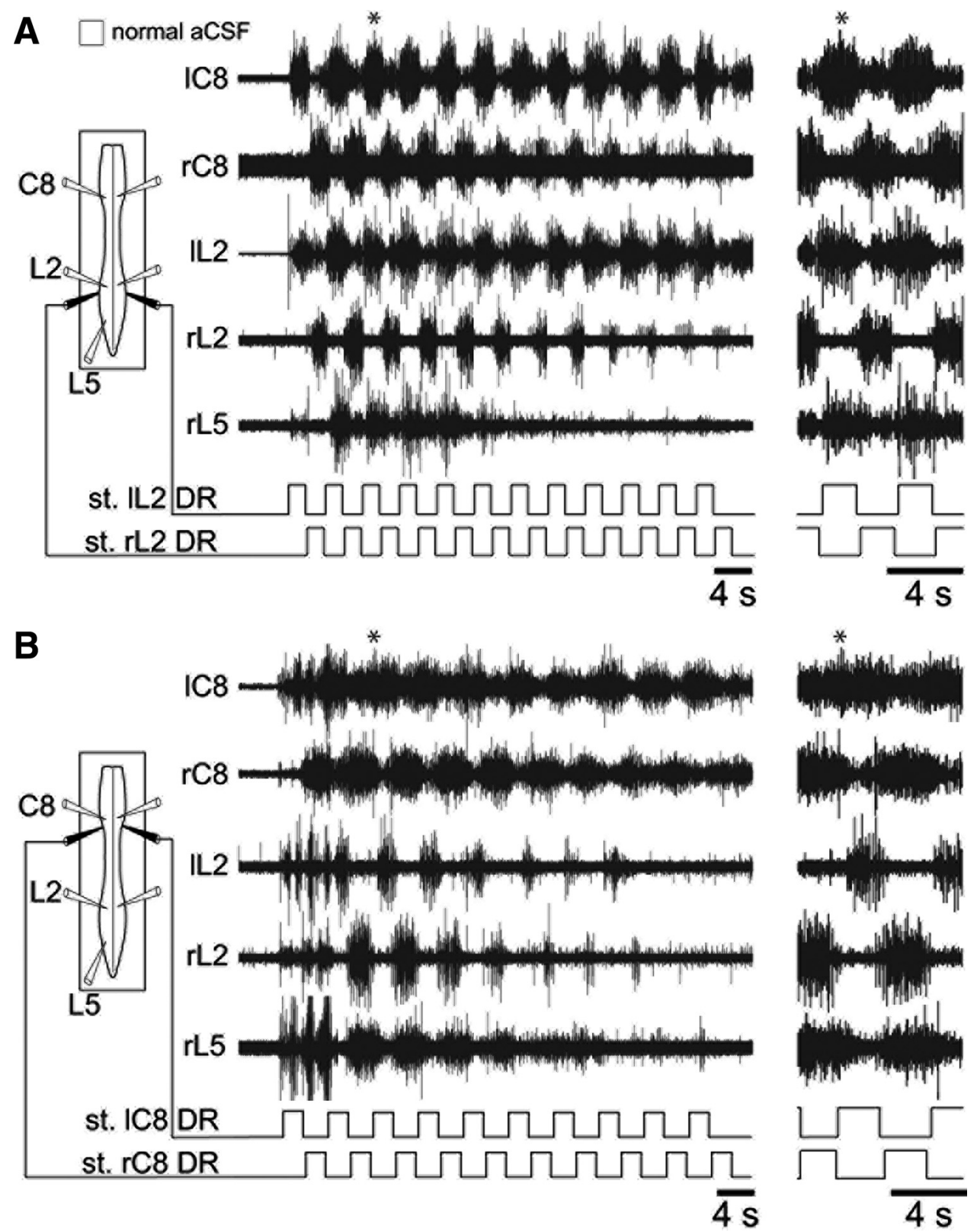

Figure 7. Coordinated cervicolumbar ventral root burst activation by alternating bilateral stimulation (st.) of low-threshold lumbar or cervical dorsal root afferents. $\boldsymbol{A}, \boldsymbol{B}$, Left, Schematics of experimental procedure. Middle, Raw motor activity at lumbar (left and right L2, L5) and cervical (left and right (8) levels during alternating cyclic stimulation (monitored in lower traces) of left and right L2 $(\boldsymbol{A})$ or $(8(\boldsymbol{B})$ DRs. In each case, an expanded portion (indicated by asterisks) is placed at right to show greater detail. Note the occurrence of alternating $(\boldsymbol{A})$ or overlapping $(\boldsymbol{B}) \mathrm{L} 2$ and $L 5$ motor bursts during the lumbar or cervical DR stimulation, respectively.

during CPG activation by L2 DR stimulation (Fig. 6B2, right polar plot, phase diagram; $\Phi=0.57$ ). However, during anteroposterior CPG activation by C8 DR stimulation (Fig. 6C2, right polar plot; $\Phi=0.17)$, L5 motor bursts occurred relatively earlier in each L2 burst cycle, resulting in a substantial overlap in the discharge of these functionally antagonistic motor pools (Fig. $6 C 2$, right, phase diagram).

A possible explanation for this unexpected, albeit consistent, alteration in the relative timing of lumbar flexor and extensor motor bursts in response to cervical afferent pathway activation was that it resulted from the unilateral mode of DR stimulation. Because peripheral sensory inputs from the left and right limb pairs are activated in phase opposition during actual stepping (Nieuwenhuijzen et al., 2000; Pearson, 2000), an additional series of experiments was conducted in which low-threshold DR affer- ents to both sides of the lumbar or cervical cord were stimulated alternately to more accurately reproduce the bilateral patterning of proprioceptive information input that occurs in vivo. However, in close correspondence with the effects of solitary DR activation (Fig, $5 B, C$ ), a strictly antiphase relationship or an overall tendency toward coincident bursting continued to be expressed by homolateral flexor L 2 and extensor L 5 ventral roots in activity patterns elicited by alternating electrical stimulation of left and right L2 (Fig. $7 A ; n=9$ preparations) or $\mathrm{C} 8$ (Fig. $7 B ; n=7$ ) dorsal roots, respectively. Here again, in the two experimental situations, robust bilaterally alternating bursting persisted at the two spinal enlargements, and with homolateral C8 and L2 burst discharge displaying an in-phase coordination. Thus, the anteroposterior and flexor/extensor coupling within the rhythmic motor patterns generated by unilateral or alternating bilateral activation 
A1

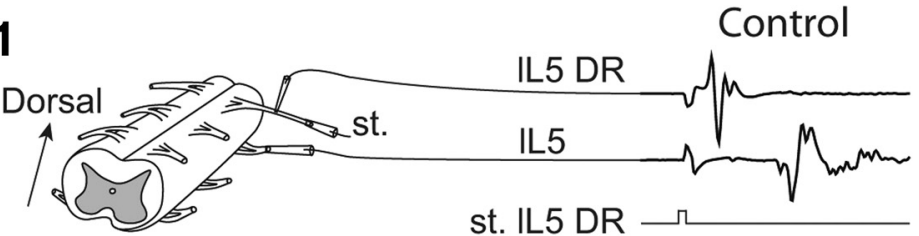

A2

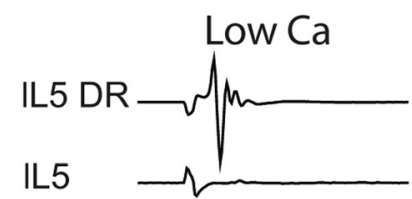

A3



B

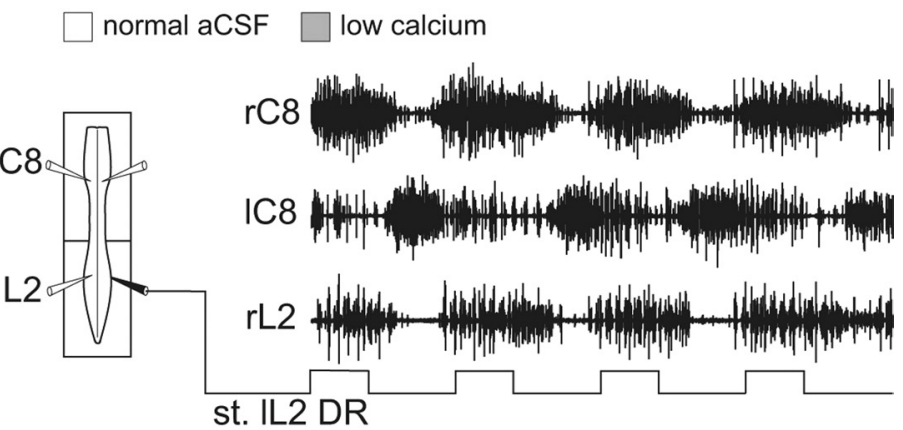

C

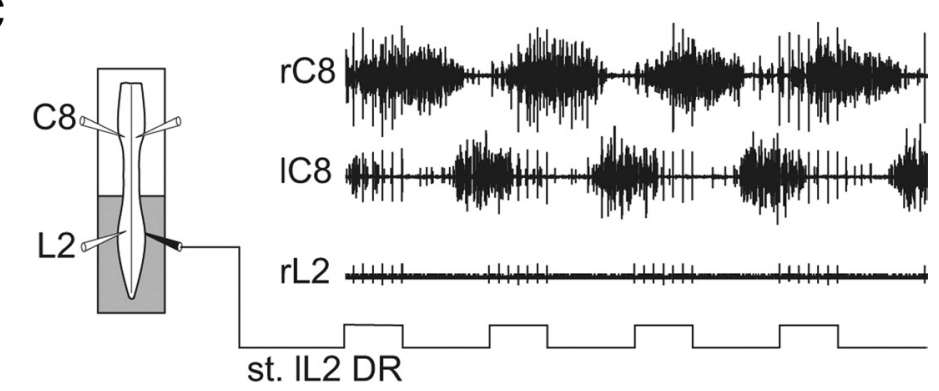

D

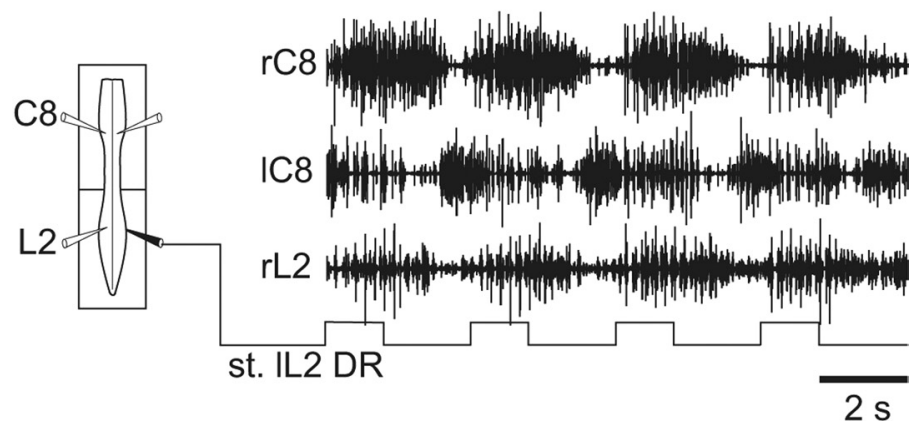

Figure 8. Direct ascending influence of low-threshold lumbar sensory afferents on the cervical pattern generators. $\mathbf{A 1}$-A3, Ability of low-calcium ACSF to reversibly block synaptic transmission in segmental reflex pathways. A1, A low-threshold afferent volley (top trace) recorded proximally from an $L 5$ DR in response to a distally applied stimulus (st.) pulse ( $0.6 \mathrm{~V}$; bottom trace) and the synaptically driven response in the $\mathrm{L} 5$ ventral root (middle trace). In the presence of low $\mathrm{Ca}^{2+} / \mathrm{high}^{\mathrm{Mg}}{ }^{2+}(\boldsymbol{A} 2)$, the motor response to the same DR activation was abolished, but reappeared after washout with normal ACSF (A3). $\boldsymbol{B}-\boldsymbol{D}$, Left, Schematics of the isolated spinal cord with locations of recording electrodes (unfilled) placed on cervical (left and right (8) and lumbar (right L2) ventral roots, and a stimulating electrode (filled) placed on the left L2 DR. A Vaseline partition allowed the differential perfusion of the cervical and lumbar cord regions. Right, Effects of cyclic L2 DR activation on bilateral C8 and right L2 ventral root activity under

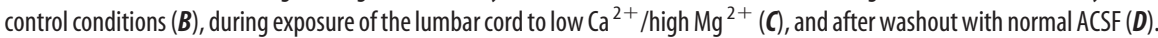
Robust bilaterally alternating cervical bursting persisted in response to L2 DR stimulation under the two perfusion conditions and notably in the absence of lumbar (PG activity (as in C). of cervical or lumbar afferent inputs are closely comparable. Furthermore, together these results indicated that the alterations in motor burst coordination expressed during the different modes of forelimb afferent pathway activation derive from changes in the flexor/extensor phase relationships within the distant hindlimb generators themselves rather than by en bloc changes in cervicolumbar CPG coupling.

Intervention of CPG circuitry in the coordinating influences of limb afferent inputs

The remote triggering action of lumbar (L2) sensory afferents on the forelimb locomotory CPGs, for example, could be conveyed by direct neuronal pathways that project up the spinal cord, or the input signals may be transmitted indirectly through a local activation of the lumbar generator circuits that in turn activate their cervical counterparts via secondorder propriospinal pathways. To distinguish between these two possibilities, L2 DR stimulation was performed in isolated preparations under conditions in which any synaptic input from L2 afferent fibers to the lumbar CPG networks was reversibly blocked with low $\mathrm{Ca}^{2+} /$ high $\mathrm{Mg}^{2+}$ ACSF applied selectively to the lumbosacral cord.

The initial verification that low $\mathrm{Ca}^{2+}$ ACSF indeed suppresses synaptic transmission in the spinal cord is illustrated in the test experiment $(n=3)$ of Figure $8 A$, where afferent fiber impulses were elicited by pulse stimulation $(0.6 \mathrm{~V} ; 1.5$ times $\mathrm{T})$ applied more distally to a lumbar (L5) DR while monosynaptic reflex responses were recorded in the corresponding L5 ventral root (Fig. $8 \mathrm{A1}$ ). With a similar DR stimulation in the presence of reduced $\mathrm{Ca}^{2+}$ ACSF (Fig. 8A2), although the resulting low-threshold afferent volley recorded more proximally remained unaffected, the ventral root response had disappeared, indicating an effective synaptic blockade in the segmental sensory-motor circuitry (Strauss and Lev-Tov, 2002). Moreover, this reflex pathway blockade, which persisted even with stronger DR stimulus intensities (up to four times T), was removed by washout with normal ACSF (Fig. 8A3).

Under normal ACSF conditions in subsequent experiments $(n=4)$ in which DR train stimulation was now applied, the rhythmic activation of a single L2 DR (pulse intensity 1.5 times T; duration, 0.3 $\mathrm{ms}$; frequency, $5 \mathrm{~Hz}$; train period of 2-4s) elicited coordinated locomotor-like activ- 
ity in cervical and lumbar segments as described above (Figs. $4 A 1,8 B$ ). When the posterior cord region was exposed to a low $\mathrm{Ca}^{2+} /$ high $\mathrm{Mg}^{2+}$ solution (Fig. $8 C$ ), the same lumbar afferent stimulation continued to elicit robust, bilaterally alternating bursting in cervical ventral roots, whereas, consistent with the synaptic blockade of segmental reflex pathways seen in Figure $8 A 2$, its ability to induce lumbar locomotor-related activity was suppressed (Fig. 8C, L2 trace). Again, this blockade of lumbar CPG operation was restored by replacing the modified bathing solution in the posterior compartment with normal ACSF (Fig. $8 D$ ). The maintenance of lumbar afferent-driven cervical rhythmicity in the absence of lumbar CPG activation (Fig. 8C) therefore strongly suggested that L2 sensory afferents have direct access to the cervical CPG circuitry via projection pathways that bypass the lumbar locomotor generators and that are likely to participate in forelimb/hindlimb coordination during actual locomotion.

In a series of corollary experiments $(n=4)$, the cervical C8 DR of one side was stimulated rhythmically while the cervical cord region was in turn subjected selectively to synaptic blockade by low $\mathrm{Ca}^{2+} /$ high $\mathrm{Mg}^{2+}$ exposure. As seen previously (Fig. 4A2), under control ACSF conditions, stimulation of low-threshold C8 DR afferents induced rhythmic motor bursting in both cervical and lumbar ventral roots (Fig. 9A). However, the application of the low $\mathrm{Ca}^{2+}$ solution to the anterior cord now prevented the expression of the cervical afferent-induced bursting at both the cervical and lumbar levels (Fig. 9B). This absence of lumbar CPG activation was not somehow due to a diminished viability of the preparation, since under the same differential bathing conditions, cyclic electrical stimulation of a lumbar L2 DR was able to elicit alternating bursting in the left and right L2 motor roots (Fig. 9C). Finally, when normal ACSF was reapplied to the entire cord, stimulation of the C8 DR again induced coordinated, bilaterally alternating rhythmicity at both the cervical and lumbar cord levels (Fig. 9D). These results therefore lead to the conclusion that, in contrast to the predominantly direct access of lumbar sensory afferents to the cervical CPG, a substantial component of the descending coordinating influence of cervical sensory inputs on the lumbar pattern generators requires the active intervention of cervical CPG circuitry.

\section{Discussion}

The findings of this study, which are summarized in Figure 10, provide new evidence for the functional organization of thoracic cord relay circuitry and its role in the coordination of the cervical and lumbar CPGs in the neonatal rat spinal cord. We also demonstrate that the activation of proprioceptive input pathways arising from either the forelimbs or hindlimbs is capable of eliciting sustained episodes of coordinated locomotor-like activity at both cervical and lumbar cord levels. Furthermore, our data show that this coupled anteroposterior bursting evoked by limb afferent stimulation is accomplished by an asymmetric ascending and descending control of the distant cervical and lumbar CPG networks by hindlimb and forelimb sensory pathways, respectively.

\section{Role of thoracic circuitry in cervicolumbar CPG coupling}

Locomotor-related activity generated in vitro by the neurochemically stimulated spinal cord in isolation from supraspinal influences and movement-related sensory feedback occurs in a stereotyped coordination pattern that corresponds to fictive quadrupedal walking (Juvin et al., 2005, 2007). Moreover, the propriospinal coupling between the lumbar and cervical CPGs was found previously to be mediated predominantly by asymmetric
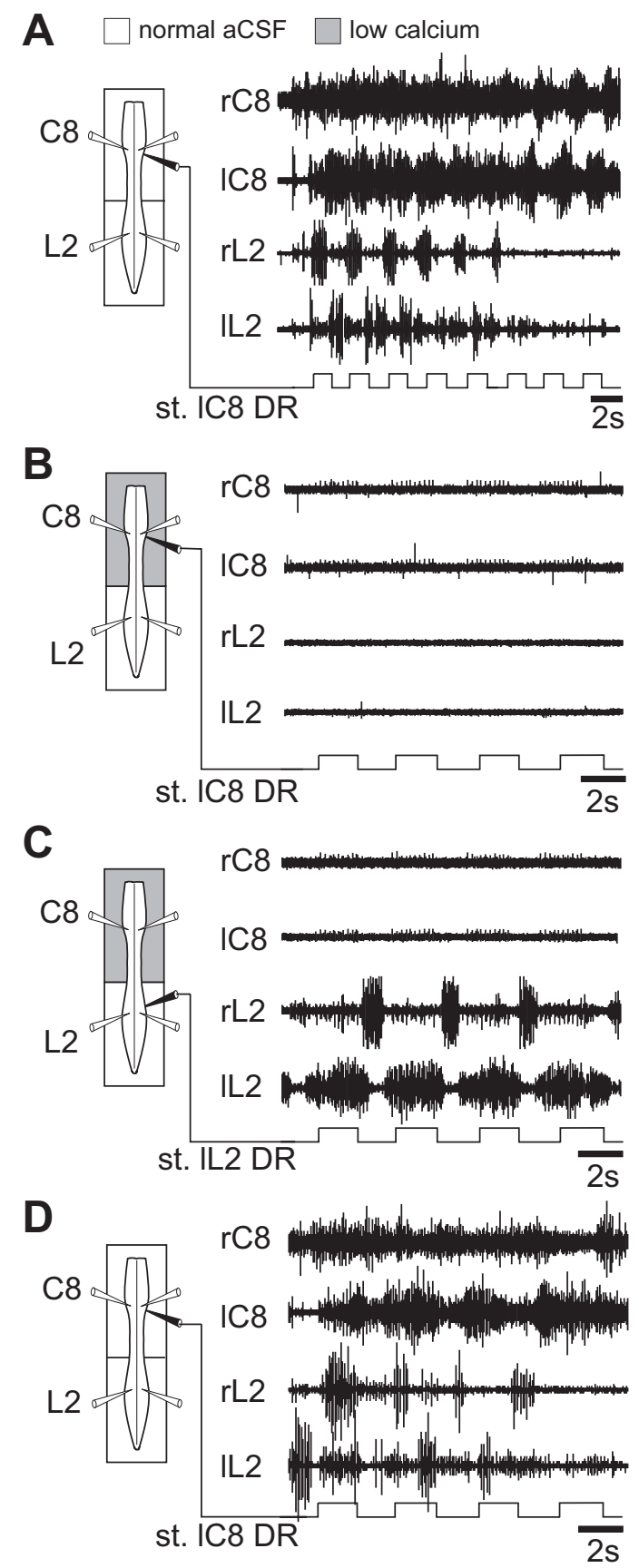

Figure 9. The descending influence of low-threshold cervical afferent inputs on the lumbar locomotor CPGs requires cervical pattern generator activation. $A-D$, Left, Schematics of isolated preparations showing recording electrode locations (left/right $(8$ and $L 2$ ventral roots) and a stimulating electrode placed on either the left $C 8(\boldsymbol{A}, \boldsymbol{B}, \boldsymbol{D})$ or left L2 DRs ( $\boldsymbol{C}$. A Vaseline partition allowed the differential perfusion of the cervical and lumbar cord regions. Right, Effects of cyclic stimulation (st.) of low-threshold cervical afferents on bilateral 88 and L2 motor root activity under control conditions $(\boldsymbol{A})$, during blocked of synaptic transmission in the cervical cord region

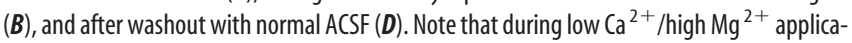
tion to the anterior cord, cyclic stimulation of an L2 DR was still able to activate bilaterally alternating bursting in $\mathrm{L} 2$ motor roots $(\boldsymbol{C})$.

ascending influences from the hindlimb generators to their forelimb counterparts via a caudorostrally directed gradient of excitability in the intervening thoracic cord segments (Juvin et al., 2005) (Fig. 10).

Our present data confirm and add to these earlier findings on the pivotal role played by thoracic circuitry in lumbocervical CPG coor- 


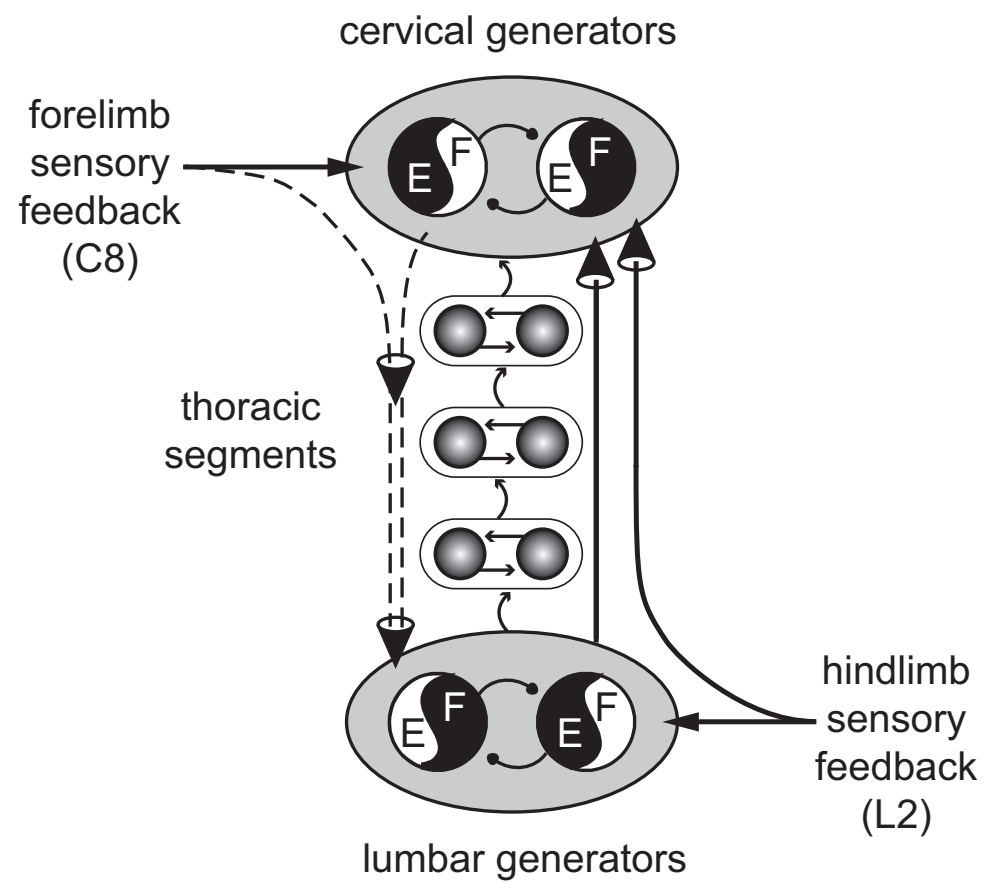

Figure 10. Summary diagram of asymmetrical spinal pathways contributing to the coordination of the spatially distant rhythmogenic networks underlying quadrupedal locomotion in neonatal rat. In addition to direct ascending inter-network connections from the hindlimb-to-forelimb pattern generators (solid vertical line at right), anteroposterior limb coordination requires the active participation of relay circuitry in intervening thoracic cord segments (shaded circles and intersegmental arrows). Moreover, unlike the ascending influence of hindlimb sensory afferents on the cervical pattern generators, which bypasses the lumbar CPGs (second vertical line at right), the rostrocaudal impact of forelimb sensory inputs on the hindlimb generators requires the concomitant activation of the cervical CPGs (dashed vertical lines at left). See Discussion for further explanation. E, Extensor; F, flexor.

dination. This was evident in partitioned-bath experiments, where the selective blockade of synaptic transmission in the thoracic cord region (Fig. 1) or withholding the latter from chemical stimulation (Fig. 2) substantially decreased the coordination between ongoing cervical and lumbar generator bursting. Thus, although long-direct ascending and descending fibers projecting in the ventrolateral funiculi are known to communicate between the cervical and lumbar enlargements (Reed et al., 2006, 2009), these through pathways are alone insufficient to ensure effective inter-CPG coordination. Rather, coupling is critically dependent on additional signal transmission through polysynaptic thoracic relays that are themselves active contributors to the coupling process. Although the thoracic cord in the neonatal rat was originally thought not to be intrinsically rhythmogenic (Kremer and Lev-Tov, 1997; Ballion et al., 2001), rhythmic activity has been reported to occur in the cervical and thoracic spinal segments when a 5-HT/NMA combination (Cowley and Schmidt, 1997; Ballion et al., 2001) is applied to this isolated supralumbar region. Furthermore, more recent evidence from chemically stimulating the completely isolated thoracic cord has disclosed an endogenous burst-generating capability that is presumed to underlie the metachronal propagation of spinal bursting activity during locomotion (Cazalets, 2005; Falgairolle and Cazalets, 2007). On this basis, thoracic circuitry can be seen to provide a variable coupling mechanism by which alterations in its inherent excitability is able to upregulate (via the expression of actively distributed bursting that is rhythmically entrained by the lumbar and/or cervical CPGs) or downregulate (as is presumably required for independent forelimb and hindlimb movements) the extent of inter-CPG communication.

The relative contribution of synaptically relayed signaling (vs transmission via long projecting pathways) to cervical and lum- bar CPG coordination bears a striking equivalence to the descending pathways that normally convey locomotor commands to these networks from the brainstem (Cowley et al., 2010). For example, blocking synaptic activity in the cervicothoracic cord suppresses lumbar locomotorrelated activity that is otherwise elicited by electrical or chemical brainstem stimulation, thereby indicating the primary involvement of propriospinal synaptic relays in mediating the descending signal transmission (Zaporozhets et al., 2004, 2006). Correspondingly, brainstem-evoked activation of the lumbar CPGs is unaffected by the staggered disruption of long ipsilateral bulbospinal projections, but the propagation of locomotory command signals is facilitated by increasing neuronal excitability in the intervening cervicothoracic region (Cowley et al., 2008). Furthermore, the persistence of effective descending signaling in the presence of contralateral cord hemisections implicated the involvement of cross-cord propriospinal pathways. Here, we find that propriospinal commissural projections also contribute substantially to the inter-CPG transmission of locomotor signals, as indicated by the significant decrease in cervicolumbar burst coordination following a midsagittal thoracic cord section (Fig. 3).

Thus, although the isolated spinal cord invariably expresses diagonally coupled bursting that corresponds to quadrupedal walking in vivo (Juvin et al., 2005, 2007), it is likely that the capacity of propriospinal circuitry to coordinate the forelimb and hindlimb generators through the intervention of both uncrossed (Juvin et al., 2005) and crossed thoracic axonal pathways provides the substrates for locomotor gait changes in the behaving animal.

\section{Fictive quadruped locomotion induced by limb sensory afferent activation}

The ability of sensory signals to activate locomotor CPG networks has long been known to enable spinalized mammals to execute hindlimb treadmill stepping (Pearson, 2004; Rossignol et al., 2006; Edgerton et al., 2008). Sensory input from hindlimb load and joint receptors is a potent activator of locomotor rhythmogenesis in spinal cord injury patients (Dietz et al., 2009) and is reportedly essential for inducing hindlimb stepping in spinal cat (Norton and Mushahwar, 2010). Moreover, the capacity of lumbar DR stimulation to activate hindlimb CPG circuitry (Smith et al., 1988; Marchetti et al., 2001) and reset/entrain ongoing lumbar rhythms (Kiehn et al., 1992; Sqalli-Houssaini et al., 1993; Iizuka et al., 1997) has been established in the isolated rodent spinal cord. However, whether sensory information from a given limb can activate the other limb-controlling generators and participate in their cycle-by-cycle coordination has hitherto remained unknown.

To our knowledge, our results show for the first time in a mammal that cyclic or tonic stimulation of low-threshold afferent pathways from a single hindlimb or forelimb is capable of driving well-coordinated patterns of rhythmic locomotor-related bursting at both lumbar and cervical cord levels (Figs. 4, 5). This 
indicates that sensory input from a given limb is not only able to influence its adjacent segmental circuitry, but also has access to the distant constitutive components of the spinal locomotor network ensemble. In parallel with such remote sensory-to-CPG influences, the cyclic modulation of reflex pathways by distant limb-controlling circuitry, which assists in coupling rhythmic arm and leg movements during human walking (Baldissera et al., 1998; Zehr et al., 2004), may also contribute to interlimb coordination during quadrupedal locomotion, as was originally proposed in cats (Miller et al., 1975). Although the identity of the receptor pathways responsible for coupled cervicolumbar CPG rhythmicity was not established in our experiments, the low stimulus intensities used to activate these inputs is compatible with the involvement of large diameter fibers, most likely proprioceptive, that are phasically active during locomotion and arise from muscle spindles or Golgi tendon organs (Kudo and Yamada, 1987; Iizuka et al., 1997; Morin and Viala, 2002).

We reported previously that chemically activated lumbar generators can drive their cervical counterparts in the absence of pharmacological stimulation at the cervical level, but not vice versa (Juvin et al., 2005), indicating a caudorostral dominance in the inter-CPG coordinating pathways (Fig. 10). Our present split-bath perfusion experiments additionally revealed a longitudinal asymmetry in the remote actions of limb proprioceptive inputs: lumbar afferent stimulation in the absence of lumbar CPG activity can elicit cervical CPG rhythmicity (Fig. 8), whereas cervical DR inputs are unable to drive the lumbar generators without concomitant cervical CPG activation (Fig. 9). This indicated that the essential activating influence of lumbar sensory inputs on the cervical generators is conveyed via direct pathways that bypass the lumbar CPGs themselves, whereas the latter are driven by cervical sensory inputs that require a summation of signals carried by both direct and indirect (via cervical CPG activation) caudally directed pathways (Fig. 10). Presumably this asymmetric remote control by extraspinal sensory inputs, which parallels the predominately ascending propriospinal drive that couples the lumbar and cervical generators (Juvin et al., 2005), serves to reinforce and stabilize the performance of the limb CPGs during actual locomotion (Hultborn and Nielsen, 2007), while ensuring that the motor patterns produced by these spatially distant networks are rapidly coadapted to changing environmental demands.

We also found that the remote activation of hindlimb CPG circuitry in vitro by forelimb sensory pathway stimulation elicits lumbar ventral root bursting in a coordination pattern that differed from the typically alternating flexor/extensor activity observed under neurochemical activation (Kjaerulff and Kiehn, 1996; Cowley and Schmidt, 1997) or in response to lumbar afferent stimulation (Marchetti et al., 2001). The overlapping lumbar flexor/extensor burst pattern evoked by either rhythmic unilateral (Fig. 6) or alternating bilateral (Fig. 7) cervical DR activation therefore points to a further asymmetry in the sensory pathway influences projecting upward or downward in the spinal cord. This may arise from differences in the way in which these afferent systems and their spinal interneuronal targets are activated by electrical DR stimulation in our in vitro experimental conditions (Klein et al., 2010). Alternatively, the asymmetry may reflect anteroposterior differences in the intrinsic design of the interneuronal systems themselves such that descending or ascending afferent signals are conveyed respectively to different cellular levels of the remote lumbar or cervical CPG networks (McCrea and Rybak, 2008). However, the possibility that the asymmetric responsiveness might be related to the particular DRs selected for stimulation in these experiments cannot be completely excluded. Although C8 and L2 DRs were chosen because of their presumed privileged access to the forelimb and hindlimb locomotor networks, respectively, further studies will be required to evaluate the ability of different spinal DRs (i.e., flexor- vs extensordominant roots at the cervical, thoracic, and lumbosacral levels) to directly or indirectly activate distant CPG circuitry.

Finally, since the restoration of locomotor function after spinal lesions is likely to involve multiple plasticity processes (Rossignol, 2000), including a remodeling both of propriospinal circuitry (Bareyre et al., 2004) and the sensorimotor pathways that process limb movement (Edgerton et al., 2008), understanding the intraspinal and extraspinal mechanisms that normally contribute to coordinated quadrupedal movements could provide important insights for spinal cord injury rehabilitation.

\section{References}

Baldissera F, Cavallari P, Leocani L (1998) Cyclic modulation of the H-reflex in a wrist flexor during rhythmic flexion-extension movements of the ipsilateral foot. Exp Brain Res 118:427-430.

Ballion B, Morin D, Viala D (2001) Forelimb locomotor generators and quadrupedal locomotion in the neonatal rat. Eur J Neurosci 14:1727-1738.

Bareyre FM, Kerschensteiner M, Raineteau O, Mettenleiter TC, Weinmann O, Schwab ME (2004) The injured spinal cord spontaneously forms a new intraspinal circuit in adult rats. Nat Neurosci 7:269-277.

Barriere G, Mellen N, Cazalets JR (2004) Neuromodulation of the locomotor network by dopamine in the isolated spinal cord of newborn rat. Eur J Neurosci 19:1325-1335.

Cazalets JR (2005) Metachronal propagation of motoneurone burst activation in isolated spinal cord of newborn rat. J Physiol 568:583-597.

Cowley KC, Schmidt BJ (1997) Regional distribution of the locomotor pattern-generating network in the neonatal rat spinal cord. J Neurophysiol 77:247-259.

Cowley KC, Zaporozhets E, Schmidt BJ (2008) Propriospinal neurons are sufficient for bulbospinal transmission of the locomotor command signal in the neonatal rat spinal cord. J Physiol 586:1623-1635.

Cowley KC, Zaporozhets E, Joundi RA, Schmidt BJ (2009) Contribution of commissural projections to bulbospinal activation of locomotion in the in vitro neonatal rat spinal cord. J Neurophysiol 101:1171-1178.

Cowley KC, Zaporozhets E, Schmidt BJ (2010) Propriospinal transmission of the locomotor command signal in the neonatal rat. Ann N Y Acad Sci 1198:42-53

Dietz V, Grillner S, Trepp A, Hubli M, Bolliger M (2009) Changes in spinal reflex and locomotor activity after a complete spinal cord injury: a common mechanism? Brain 132:2196-2205.

Edgerton VR, Courtine G, Gerasimenko YP, Lavrov I, Ichiyama RM, Fong AJ, Cai LL, Otoshi CK, Tillakaratne NJ, Burdick JW, Roy RR (2008) Training locomotor networks. Brain Res Rev 57:241-254.

English AW (1979) Interlimb coordination during stepping in the cat: an electromyographic analysis. J Neurophysiol 42:229-243.

English AW, Weeks O (1989) Electromyographic cross-talk within a compartmentalized muscle of the cat. J Physiol 416:327-336.

Falgairolle M, Cazalets JR (2007) Metachronal coupling between spinal neuronal networks during locomotor activity in newborn rat. J Physiol 580:87-102.

Giraudin A, Cabirol-Pol MJ, Simmers J, Morin D (2008) Intercostal and abdominal respiratory motoneurons in the neonatal rat spinal cord: spatiotemporal organization and responses to limb afferent stimulation. J Neurophysiol 99:2626-2640.

Grillner S (1981) Control of locomotion in bipeds, tetrapods, and fish. In: Handbook of Physiology, the nervous system, motor control [Suppl 2], pp 1179-1236. Bethesda, MD: American Physiological Society.

Hultborn H, Nielsen JB (2007) Spinal control of locomotion-from cat to man. Acta Physiol 189:111-121.

Iizuka M, Kiehn O, Kudo N (1997) Development in neonatal rats of the sensory resetting of the locomotor rhythm induced by NMDA and 5-HT. Exp Brain Res 114:193-204.

Juvin L, Simmers J, Morin D (2005) Propriospinal circuitry underlying in- 
terlimb coordination in mammalian quadrupedal locomotion. J Neurosci 25:6025-6035.

Juvin L, Simmers J, Morin D (2007) Locomotor rhythmogenesis in the isolated rat spinal cord: a phase-coupled set of symmetrical flexion extension oscillators. J Physiol 583:115-128.

Kiehn O, Iizuka M, Kudo N (1992) Resetting from low threshold afferents of N-methyl-D-aspartate-induced locomotor rhythm in the isolated spinal cord-hindlimb preparation from newborn rats. Neurosci Lett 148:43-46.

Kjaerulff O, Kiehn O (1996) Distribution of networks generating and coordinating locomotor activity in the neonatal rat spinal cord in vitro: a lesion study. J Neurosci 16:5777-5794.

Klein DA, Patino A, Tresch MC (2010) Flexibility of motor pattern generation across stimulation conditions by the neonatal rat spinal cord. J Neurophysiol 103:1580-1590.

Kremer E, Lev-Tov A (1997) Localization of the spinal network associated with generation of hindlimb locomotion in the neonatal rat and organization of its transverse coupling system. J Neurophysiol 77:1155-1170.

Kudo N, Yamada T (1987) Morphological and physiological studies of development of the monosynaptic reflex pathway in the rat lumbar spinal cord. J Physiol 389:441-459.

Marchetti C, Beato M, Nistri A (2001) Alternating rhythmic activity induced by dorsal root stimulation in the neonatal rat spinal cord in vitro. J Physiol 530:105-112.

Matsukawa K, Kamei H, Minoda K, Udo M (1982) Interlimb coordination in cat locomotion investigated with perturbation. I. Behavioral and electromyographic study on symmetric limbs of decerebrate and awake walking cats. Exp Brain Res 46:425-437.

McCrea DA, Rybak IA (2008) Organization of mammalian locomotor rhythm and pattern generation. Brain Res Rev 57:134-146.

McKenna JE, Prusky GT, Whishaw IQ (2000) Cervical motoneuron topography reflects the proximodistal organization of muscles and movements of the rat forelimb: a retrograde carbocyanine dye analysis. J Comp Neurol 419:286-296.

Miller S, Van Der Burg J, Van Der Meche F (1975) Coordination of movements of the hindlimbs and forelimbs in different forms of locomotion in normal and decerebrate cats. Brain Res 91:217-237.

Morin D, Viala D (2002) Coordinations of locomotor and respiratory rhythms in vitro are critically dependent on hindlimb sensory inputs. J Neurosci 22:4756-4765.

Nieuwenhuijzen PH, Schillings AM, Van Galen GP, Duysens J (2000) Modulation of the startle response during human gait. J Neurophysiol 84:65-74.

Norton JA, Mushahwar VK (2010) Afferent inputs to mid- and lowerlumbar spinal segments are necessary for stepping in spinal cats. Ann N Y Acad Sci 1198:10-20.

Orlovsky GN, Deliagina TG, Grillner S (1999) Neuronal control of locomotion: from mollusc to man. New York: Oxford UP.
Pearson KG (2000) Neural adaptation in the generation of rhythmic behavior. Annu Rev Physiol 62:723-753.

Pearson KG (2004) Generating the walking gait: role of sensory feedback. Prog Brain Res 143:123-129.

Reed WR, Shum-Siu A, Onifer SM, Magnuson DS (2006) Inter-enlargement pathways in the ventrolateral funiculus of the adult rat spinal cord. Neuroscience 142:1195-1207.

Reed WR, Shum-Siu A, Whelan A, Onifer SM, Magnuson DS (2009) Anterograde labeling of ventrolateral funiculus pathways with spinal enlargement connections in the adult rat spinal cord. Brain Res 1302:76-84.

Rossignol S (1996) Neural control of stereotypic limb movements. In: Handbook of physiology, Section 12, pp 173-215. New York: Oxford UP.

Rossignol S (2000) Locomotion and its recovery after spinal injury. Curr Opin Neurobiol 10:708-716.

Rossignol S, Saltiel P, Perreault MC, Drew T, Pearson K, Belanger M (1993) Intralimb and interlimb coordination in the cat during real and fictive rhythmic motor programs. Seminars Neurosci 5:67-75.

Rossignol S, Dubuc R, Gossard JP (2006) Dynamic sensorimotor interactions in locomotion. Physiol Rev 86:89-154.

Sherrington CS (1910) Flexion-reflex of the limb, crossed extension reflex stepping and standing. J Physiol 40:28-121.

Sherrington CS (1917) Reflexes elicitable in the cat from pinna vibrissae and jaws. J Physiol 51:404-431.

Smith JC, Feldman JL, Schmidt BJ (1988) Neural mechanisms generating locomotion studied in mammalian brain stem-spinal cord in vitro. FASEB J 2:2283-2288.

Sqalli-Houssaini Y, Cazalets JR, Clarac F (1993) Oscillatory properties of the central pattern generator for locomotion in neonatal rats. J Neurophysiol 70:803-813.

Strauss I, Lev-Tov A (2002) Neural pathways between sacrocaudal afferents and lumbar pattern generators in neonatal rats. J Neurophysiol 89:773-784

Udo M, Kamei H, Matsukawa K, Tanaka K (1982) Interlimb coordination in cat locomotion investigated with perturbation. II. Correlates in neuronal activity of Deiter's cells of decerebrate walking cats. Exp Brain Res 46:438-447.

Whelan P, Bonnot A, O’Donovan MJ (2000) Properties of rhythmic activity generated by the isolated spinal cord of the neonatal mouse. J Neurophysiol 84:2821-2833.

Zaporozhets E, Cowley KC, Schmidt BJ (2004) A reliable technique for the induction of locomotor-like activity in the in vitro neonatal rat spinal cord using brainstem electrical stimulation. J Neurosci Methods 139:33-41.

Zaporozhets E, Cowley KC, Schmidt BJ (2006) Propriospinal neurons contribute to bulbospinal transmission of the locomotor command signal in the neonatal rat spinal cord. J Physiol 572:443-458.

Zehr EP, Frigon A, Hoogenboom N, Collins DF (2004) Facilitation of soleus H-reflex amplitude evoked by cutaneous nerve stimulation at the wrist is not suppressed by rhythmic arm movement. Exp Brain Res 159:382-388. 